Leonid Makar-Limanov · Jie-Tai Yu

\title{
Degree estimate for subalgebras generated by two elements
}

Received November 12, 2006 and in revised form February 12, 2007

\begin{abstract}
We develop a new combinatorial method to deal with a degree estimate for subalgebras generated by two elements in different environments. We obtain a lower bound for the degree of the elements in two-generated subalgebras of a free associative algebra over a field of zero characteristic. We also reproduce a somewhat refined degree estimate of Shestakov and Umirbaev for the polynomial algebra, which plays an essential role in the recent celebrated solution of the Nagata conjecture and the strong Nagata conjecture.
\end{abstract}

Keywords. Degree estimate, two-generated subalgebras, polynomial algebras, free associative algebras, commutators, Jacobians

\section{Introduction and main results}

Suppose $A$ is an algebra on which a degree function with the usual properties is defined. Let $B$ be a subalgebra of $A$ which is generated by two elements $f$ and $g$.

The question of what are the possible degrees for the elements of $B$ seems to be both natural and interesting. To be more specific, regarding $P \in B$ as a polynomial in $f$ and $g$, what can we say about the degree of $P(f, g)$ in terms of the degrees of $P$ relative to $f$ and $g$ ? It is rather clear that the upper bound for the degree of $P(f, g)$ can be easily found, but is it possible to find a meaningful lower bound?

The question is motivated by a result in [13] where a lower bound is discovered when $A$ is a polynomial ring over a field of zero characteristic, and $f, g$ are algebraically independent. This result plays a crucial role in the recent celebrated solution of the Nagata conjecture [14] and the strong Nagata conjecture [17].

The cases when $f$ and $g$ are algebraically dependent and algebraically independent are apparently quite different. Say, even when $A$ is a polynomial algebra in one variable (then, of course $f$ and $g$ are algebraically dependent) there is no useful estimate known to us of the smallest degree of non-constant polynomials in $B$, and this question is

L. Makar-Limanov: Department of Mathematics, Wayne State University, Detroit, MI 48202, U.S.A.; e-mail: lml@math.wayne.edu

J.-T. Yu: Department of Mathematics, The University of Hong Kong, Hong Kong SAR, China; e-mail: yujt@hkucc.hku.hk,yujietai@yahoo.com

Mathematics Subject Classification (2000): Primary 13S10, 16S10; Secondary 13F20, 13W20, 14R10, 16W20, $16 \mathrm{Z} 05$ 
very interesting indeed. For instance the well-known AMS Theorem (Abhyankar's highschool lemma, [1, 16]), is equivalent to the statement that when this degree is one, then $\min \{\operatorname{deg}(f), \operatorname{deg}(g)\} \operatorname{divides} \max \{\operatorname{deg}(f), \operatorname{deg}(g)\}$.

The degree estimate in [13] depends linearly on $\operatorname{deg}_{f}(P)$ and it seems that with the right assumptions this should be so in far greater generality. We replace $\operatorname{deg}_{f}(P)$ by the "weighted degree" of $P$. As the reader will see it is more natural to use the weighted degree instead of the degree relative to $f$. In this paper we develop a new combinatorial method based on the Lemma on radicals (below), which can be used in different environments. In particular, by this method we obtain a sharp degree estimate for the "free" case, namely when $A$ is either a free associative algebra or a polynomial algebra over a field of zero characteristic. For the free associative algebra our degree estimate is new. For the polynomial case we reproduce the degree estimate of Shestakov and Umirbaev (see [13]) with some improvement.

We are going to treat both cases more or less in parallel. In order to formulate the results let us introduce necessary notions and notations. Below $A$ stands for either a polynomial algebra or a free associative algebra.

From our discussion above it is clear that for the polynomial case it is essential to assume that $f$ and $g$ are algebraically independent. We also want to assume something similar for the free case. So let us call two elements of a free algebra algebraically independent if the subalgebra generated by these two elements is a free algebra of rank two. Otherwise we will call them algebraically dependent. (We remind the reader that when two elements of a free associative algebra are algebraically dependent, then they commute and even belong to a subalgebra which is isomorphic to a free algebra of rank one, or in other words to a polynomial algebra in one variable; see [2].) Let us denote by $\operatorname{deg}(a)$ the homogeneous (total) degree of an element $a \in A$ and by $a^{+}$the highest homogeneous form of $a$. Let $B$ be an algebra of rank two (either polynomial or free) with generators $u$ and $v$. Let us denote by $\operatorname{deg}_{u}(b)$ and $\operatorname{deg}_{v}(b)$ the degree of $b \in B$ relative to $u$ and $v$ respectively. If we assign weights $\rho$ and $\sigma$ to $u$ and $v$ respectively we can define a weighted degree function $w_{\rho, \sigma}$ on $B$ as follows. If $\mu \in B$ is a monomial then $w_{\rho, \sigma}(\mu)=$ $\rho \operatorname{deg}_{u}(\mu)+\sigma \operatorname{deg}_{v}(\mu)$ and $w_{\rho, \sigma}(b)=\max \left(w_{\rho, \sigma}(\mu)\right)$ over all monomials $\mu$ appearing in $b$ with a non-zero coefficient. (In this notation $\operatorname{deg}_{u}=\operatorname{deg}_{1,0}$ and $\operatorname{deg}_{v}=\operatorname{deg}_{0,1}$.) It is clear that $w_{\operatorname{deg}(f), \operatorname{deg}(g)}(P)$ gives an upper bound for $\operatorname{deg}(P(f, g))$. Of course, if $f^{+}$ and $g^{+}$are algebraically independent then $\operatorname{deg}(P(f, g))=w_{\operatorname{deg}(f), \operatorname{deg}(g)}(P)$.

Finally, let $[f, g]=f g-g f$ denote the commutator of the elements $f, g \in A$ (free case) and let

$$
d f \wedge d g=\sum\left(\frac{\partial f}{\partial x_{i}} \frac{\partial g}{\partial x_{j}}-\frac{\partial f}{\partial x_{j}} \frac{\partial g}{\partial x_{i}}\right) d x_{i} \wedge d x_{j}
$$

denote the corresponding differential 2-form (commutative case).

Here is our main result:

Theorem 1.1. Let $A=F\left\langle x_{1}, \ldots, x_{k}\right\rangle$ be a free associative algebra over a field $F$ of zero characteristic and let $f, g \in A$ be algebraically independent. Suppose that $f^{+}$and $g^{+}$are algebraically dependent and neither $\operatorname{deg}(f)$ divides $\operatorname{deg}(g)$ nor $\operatorname{deg}(g)$ divides $\operatorname{deg}(f)$. 
Then for any $P \in F\langle x, y\rangle$ we have

$$
\operatorname{deg}(P(f, g)) \geq D(f, g) w_{\operatorname{deg}(f), \operatorname{deg}(g)}(P) \quad \text { where } \quad D(f, g)=\frac{\operatorname{deg}([f, g])}{\operatorname{deg}(f g)} .
$$

We also reproduce the degree estimate obtained in [13].

Proposition 1.2. Let $A=F\left[x_{1}, \ldots, x_{k}\right]$ be a polynomial algebra over a field $F$ of zero characteristic, $f, g \in A$ be algebraically independent, and $P \in F[x, y]$. Then

$$
\operatorname{deg}(P(f, g)) \geq D(f, g) w_{\operatorname{deg}(f), \operatorname{deg}(g)}(P)
$$

where

$$
D(f, g)=\left[1-\frac{(\operatorname{deg}(f), \operatorname{deg}(g))(\operatorname{deg}(f g)-\operatorname{deg}(d f \wedge d g))}{\operatorname{deg}(f) \operatorname{deg}(g)}\right] .
$$

Here $(\operatorname{deg}(f), \operatorname{deg}(g))$ is the greatest common divisor of $\operatorname{deg}(f)$ and $\operatorname{deg}(g)$, and $\operatorname{deg}(d f \wedge d g)$ is the degree of the differential form $d f \wedge d g$, i.e. it is 2 plus the largest of the degrees of the 2 by 2 minors of the Jacobi matrix of $f$ and $g$ with respect to $x_{1}, \ldots, x_{k}$.

Remark 1.3. 1) Both estimates are sharp. Indeed, take two relatively prime natural numbers $m$ and $n$ and consider $f=x^{n}$ and $g=x^{m}+y$. Let $P(f, g)=[f, g]$ in the free case and $P(f, g)=g^{n}-f^{m}$ in the polynomial case. For these polynomials both estimates become equalities.

2) The estimate in [13] follows from the estimate of Proposition 1.2 since $P(f, g)$ contains a monomial $f^{\operatorname{deg}_{1}(P)} g^{j}$ where $j \geq 0$; hence

$$
w_{\operatorname{deg}(f), \operatorname{deg}(g)}(P(f, g)) \geq \operatorname{deg}_{1}(P) \operatorname{deg}(f) ;
$$

replacing $w_{\operatorname{deg}(f), \operatorname{deg}(g)}(P)$ by $\operatorname{deg}_{1}(P) \operatorname{deg}(f)$ one gets the estimate from [13]. Also in [13] it is required that $(f, g)$ is a so-called $*$-pair, i.e. neither $f^{+}$is a power of $g^{+}$nor $g^{+}$ is a power of $f^{+}$, which we do not need in Proposition 1.2. So the estimate obtained in Proposition 1.2 can be viewed as a refinement of the estimate in [13]. In the free case we do require that $(f, g)$ is a $*$-pair (our non-divisibility condition), and the example $f=x$, $g=x^{m}+y$, and $P=g-f^{m}$ shows that this condition is essential: $1<\frac{2}{m+1} m$. See the explanation in Remark 3.2.

3) Both in Theorem 1.1 and in Proposition 1.2 the homogeneous degree can be replaced by a weighted degree defined by $w\left(x_{i}\right)=n_{i}$ where $n_{i}$ are positive integers. To obtain the corresponding formulae just substitute $y_{i}^{n_{i}}$ in place of $x_{i}$ and then use the estimates for the homogeneous degree function.

4) It seems natural to include into considerations the case when $A$ is a free Lie algebra. Unfortunately in this case as well as in the case of any free algebra $A$ with the Nielsen-Schreier property (any subalgebra of $A$ is free) algebraic independence of $f$ and $g$ implies that $f^{+}$and $g^{+}$are either algebraically independent or linearly dependent. See, for instance, Theorem 11.1.1, p. 195 in [9]. So we can always assume that $f^{+}$ and $g^{+}$are algebraically independent and then $\operatorname{deg}(P(f, g))=w_{\operatorname{deg}(f), \operatorname{deg}(g)}(P)$, i.e. no reduction in degree is possible. 


\section{Reduction by Lemma on radicals}

As the first step we extend the algebra $A$ to the algebra $\mathcal{A}$ of Mal'tsev-Neumann power series.

Here is the definition of the latter. Let $G$ be a linearly ordered group (of course the order should agree with the group operations). $\mathcal{A}$ is defined as the set of all sums $\sum_{g \in \Delta} c_{g} g$ where $g \in G, c_{g} \in F \backslash 0$, and $\Delta$ is a well-ordered subset of $G$. Both addition and multiplication are naturally defined though it requires some effort to prove that the multiplication is well-defined.

These algebras were introduced by Mal'tsev and Neumann [8, 11] in order to show that the group algebra of a free group can be embedded into a division ring. (This was a question raised by Moufang in [10].)

In our context it is more convenient to have $\Delta$ well-ordered relative to the opposite order; that is, any non-empty subset of $\Delta$ should have a largest element.

Let us start with polynomial algebras. In this case we take as $G$ the free abelian group $G$ on generators $x_{1}, \ldots, x_{k}$. The total degree can be defined on the elements of $G$ and it gives a partial order on $G$. This partial order can be refined to a linear order on $G$, say by adding lexicographic order $x_{1}>>\cdots>>x_{k}>1$.

For a free associative algebra take the free group $G$ on generators $x_{1}, \ldots, x_{k}$. The total degree can be defined on the elements of $G$ and again it gives a partial order on $G$. It is possible to refine this partial order (in many different ways) to a linear order on $G$ (so that the order agrees with the group operations), but the description of these orders is too involved, and the interested reader should consult [12, 15] for details.

The algebra $\mathcal{A}$ has a valuation $|a|=\max \{g \in \Delta(a)\}$, where $\Delta(a)$ is the support of $a$, i.e. the set of all $g$ appearing in $a$ with non-zero coefficients. Let $v(a)=c_{g} g$ where $g=|a| \in G$ and $c=c_{g} \in F$ is the coefficient of $g$ in $a$.

The Mal'tsev-Neumann algebras $([8,11])$ are, as mentioned, division algebras which satisfy the following

Lemma on radicals. If $a \in \mathcal{A}$ and the monomial $v(a)$ has a root: $v(a)=\left(c g_{1}\right)^{k}$ where $c \in F$ and $g_{1} \in G$, then there exists an $\alpha \in \mathcal{A}$ such that $a=\alpha^{k}$ if $k$ is not divisible by the characteristic of $F$.

This can be shown using "approximations" relative to the valuation function on $\mathcal{A}$. See [3] 4, 6] for a proof. The proof is similar to the proofs given in [5, 8, 11] that $\mathcal{A}$ is a division ring, that is, for every non-zero element of $\mathcal{A}$ there exists a root of degree -1 . See also [7] where further applications of this technique are given.

In case $A$ is a polynomial algebra, the lemma can be deduced from Newton's binomial theorem for general degree.

Let us return to $f$ and $g$ which we now consider as elements of $\mathcal{A}$. Put $v(f)=$ $c_{1} h_{1}$ and $v(g)=c_{2} h_{2}$ where $c_{i} \in F$ and $h_{i} \in G$. We can assume without loss of generality that $c_{1}=c_{2}=1$ since we can multiply $f$ and $g$ by non-zero elements of $F$. If $h_{1}$ and $h_{2}$ are algebraically independent then $\operatorname{deg}(P(f, g))=w_{\operatorname{deg}(f), \operatorname{deg}(g)}(P)$ since $v\left(f^{i_{1}} g^{j_{1}} \ldots f^{i_{k}} g^{j_{k}}\right)=v\left(f^{i_{1}}\right) v\left(g^{j_{1}}\right) \ldots v\left(f^{i_{k}}\right) v\left(g^{j_{k}}\right)$ by the definition of $v$. Also then 
$\operatorname{deg}([f, g])=\operatorname{deg}(f g)$ in the free case and $\operatorname{deg}(d f \wedge d g)=\operatorname{deg}(f g)$ in the commutative case, and $D(f, g)=1$ in both cases.

So from now on let us assume that $h_{1}$ and $h_{2}$ are algebraically dependent. Any subgroup of a free group (free abelian group) is free, therefore the subgroup generated by $h_{1}$ and $h_{2}$ is a free group of rank 1 and is generated by one element, say $h$. Since $h$ can be replaced by $h^{-1}$ we can assume that $h>1$. Let us also assume that $h$ is an element of $G$ without root. (Clearly in our situation any element of $G$ has maximal possible root.) So $h_{1}=h^{q}$ and $h_{2}=h^{p}$ for some integers $p$ and $q$. Both $v(f)>1$ and $v(g)>1$, so $p$ and $q$ are natural numbers.

If $F$ has zero characteristic, then by the lemma on radicals we can put $f=\tau^{q}$. Then $g=\tau^{p}+g_{1}$ where $v\left(g_{1}\right)<v(g)$. If $v\left(g_{1}\right)$ and $v(f)$ are algebraically dependent then $v\left(g_{1}\right)=c_{1} h^{p_{1}}$ where $p_{1}<p, g_{1}=c_{1} \tau^{p_{1}}+g_{2}$ where $v\left(g_{2}\right)<v\left(g_{1}\right)$ and so on. By repeating these steps we either obtain $g=\sum_{i=l}^{p} c_{i} \tau^{i}+s, c_{i} \in F$, where $v(f)$ and $v(s)$ are algebraically independent, or never stop.

Lemma on steps. The process above will stop after a finite number of steps.

Proof. After $k$ steps we will obtain $g=\sum_{i=k}^{p} c_{i} \tau^{i}+g_{k}$ where $v\left(g_{k}\right)<h^{k}$. Now, in the free case $[f, g]=\left[f, g_{k}\right]$ since $\left[f, \tau^{i}\right]=\left[\tau^{q}, \tau^{i}\right]=0$. Similarly in the commutative case take derivations $\partial_{i}$ on $\mathcal{A}$ which are given by $\partial_{i}\left(x_{j}\right)=\delta_{i, j}$ (where $\delta_{i, j}$ is the Kronecker delta). Then $\partial_{i}(f) \partial_{j}(g)-\partial_{j}(f) \partial_{i}(g)=\partial_{i}(f) \partial_{j}\left(g_{k}\right)-\partial_{j}(f) \partial_{i}\left(g_{k}\right)$ by elementary calculus rules. But $\operatorname{deg}([a, b]) \leq \operatorname{deg}(a b)$, or $\operatorname{deg}\left(\partial_{i}(a) \partial_{j}(b)-\partial_{j}(a) \partial_{i}(b)\right) \leq \operatorname{deg}(a b)-2$, for any $a, b$ in the corresponding $\mathcal{A}$. So $\operatorname{deg}\left(g_{k}\right) \geq \operatorname{deg}([f, g])-\operatorname{deg}(f)$, or $\operatorname{deg}\left(g_{k}\right) \geq$ $\operatorname{deg}(d f \wedge d g)-\operatorname{deg}(f)$ accordingly. Since in our setting $\operatorname{deg}(\tau)=\operatorname{deg}(h)>0$ and $\operatorname{deg}\left(\tau^{k}\right)=k \operatorname{deg}(\tau)$ we conclude that $k$ is bounded away from $-\infty$. This proves the lemma.

From this lemma we see that it is possible to write $g=\sum_{i=l}^{p} c_{i} \tau^{i}+s$ where $v(f)$ and $v(s)$ are algebraically independent.

Now we can find $\operatorname{deg}(s)$. Indeed, in the free case $[f, g]=[f, s]$ and $\operatorname{deg}([f, s])=$ $\operatorname{deg}(f)+\operatorname{deg}(s)$ since $v(f)$ and $v(s)$ are algebraically independent. So $\operatorname{deg}(s)=$ $\operatorname{deg}([f, g])-\operatorname{deg}(f)$.

Similarly in the commutative case $\partial_{i}(f) \partial_{j}(g)-\partial_{j}(f) \partial_{i}(g)=\partial_{i}(f) \partial_{j}(s)-\partial_{j}(f) \partial_{i}(s)$. It is also clear that if $\partial_{i}(v(f)) \partial_{j}(v(s))-\partial_{j}(v(f)) \partial_{i}(v(s))=0$ for all $i, j$ then $v(f)$ and $v(s)$ are algebraically dependent. Since that is not the case, at least one of these minors is not equal to zero and $\operatorname{deg}(s)=\operatorname{deg}(d f \wedge d g)-\operatorname{deg}(f)$.

Let us remark that in both cases $\operatorname{deg}(s) \leq \operatorname{deg}(g)$.

Consider the subalgebra $C$ of $\mathcal{A}$ which is generated by $\tau, \tau^{-1}$, and $s$. Clearly $f, g \in C$. Take the weighted degree function $w_{1, p}$ on $C$ given by $w_{1, p}(\tau)=1, w_{1, p}(s)=p$. (It is defined exactly as the weighted degree function on a free algebra.) Let $\widetilde{a}$ be the leading form of an element $a \in C$ relative to $w_{1, p}$. Then $w_{1, p}(f)=q, w_{1, p}(g)=p$, and $\widetilde{f}=\tau^{q}$, $\tilde{g}=\tau^{p}+s$

Take now any polynomial $P(x, y)$ where $x$ and $y$ are independent variables. It can be written as $P(x, y)=\bar{P}(x, y)+P_{1}(x, y)$ where $\bar{P}(x, y)$ is $q$, $p$-homogeneous, $w_{q, p}(\bar{P}(x, y))=w_{q, p}(P(x, y))$, and $w_{q, p}\left(P_{1}(x, y)\right)<w_{q, p}(P(x, y)) .(\bar{P}(x, y)$ is the leading form of $P(x, y)$ relative to $\left.w_{q, p}.\right)$ 
Lemma on degree. Let deg be the homogeneous degree on $\mathcal{A}$. Then $\operatorname{deg}(P(f, g)) \geq$ $\operatorname{deg}(\bar{P}(\widetilde{f}, \widetilde{g}))$ for any polynomial $P$.

Proof. Represent $P(f, g)$ as above: $P(f, g)=\bar{P}(f, g)+P_{1}(f, g)$. Let $P(f, g)=$ $Q\left(\tau, \tau^{-1}, s\right), P_{1}(f, g)=Q_{1}\left(\tau, \tau^{-1}, s\right)$, and $\bar{P}(\tilde{f}, \widetilde{g})=R(\tau, s)$. Since $v(\tau)=h$ and $v(s)$ are algebraically independent elements of $G$, for any polynomial $S\left(\tau, \tau^{-1}, s\right)$ its $\operatorname{deg}\left(S\left(\tau, \tau^{-1}, s\right)\right)$ is the maximum of the degrees of the monomials in $s, \tau$, and $\tau^{-1}$ appearing in $S$ with non-zero coefficients. Of course, in order to compute $\operatorname{deg}\left(S\left(\tau, \tau^{-1}, s\right)\right)$ we should substitute expressions of $s$ and $\tau$ as elements of $\mathcal{A}$. It is clear that $w_{1, p}(\mathbf{m}) \leq$ $w_{q, p}\left(P_{1}(f, g)\right)$ for any monomial $\mathbf{m}$ appearing in $Q_{1}$ with non-zero coefficient. It is also clear that $\bar{P}(f, g)=\bar{P}(\tilde{f}, \widetilde{g})+r\left(\tau, \tau^{-1}, s\right)$ where $w_{1, p}(r)<w_{q, p}(\bar{P})$. Therefore all monomials of $R$ appear in $Q$ with non-zero coefficients, which proves the lemma.

Now in order to obtain an estimate of $\operatorname{deg}(P(f, g))$ we can replace $P$ by $\bar{P}$ which is uniquely determined by $\operatorname{deg}(f)$ and $\operatorname{deg}(g)$, and further replace $f$ and $g$ by $\widetilde{f}$ and $\widetilde{g}$ which are uniquely determined by $v(f), v(g)$, and $v([f, g])$ or $v(f \wedge g)$.

We have reduced our main problem to the following question.

Take $f=\tau^{p}, g=\tau^{q}+s$ where $\tau$ and $s$ generate either a free associative or a polynomial algebra of rank two. Estimate $\operatorname{deg}(P(f, g))$ provided:

- the polynomial $P$ is $n, m$-homogeneous;

- $\operatorname{deg}(\tau)=n / p=m / q, \operatorname{deg}(s) \leq \operatorname{deg}(g)$;

- on the algebra generated by $\tau$ and $s$, the degree deg is given by $w_{\operatorname{deg}(\tau), \operatorname{deg}(s)}$.

Let us introduce $t^{e}=\tau$ where $e=n / p$. Then $f=t^{n}, g=t^{m}+s$. We replaced $\tau$ by $t$ since it is more convenient for further computations to have $\operatorname{deg}_{t}(f)=\operatorname{deg}(f)$, $\operatorname{deg}_{t}(g)=\operatorname{deg}(g)$, and $\operatorname{deg}(t)=1$.

From now on we assume that $f=t^{n}$ and $g=t^{m}+s$ and that $P(f, g)$ is $n, m$ homogeneous.

\section{Degree estimate for free associative algebras}

In this section $F\langle t, s\rangle$ is a free algebra of rank two. In view of the hypothesis of Theorem 1.1 we assume that $m>n$ and that $n$ does not divide $m$.

Lemma on monomials. Assume $w_{n, m}(P(f, g))=N$. Let $q$ be the integral part of $N /(n+m)$. Then $Q(t, s)=P\left(t^{n}, t^{m}+s\right)$ contains a monomial $\mu$ with $\operatorname{deg}_{s}(\mu) \leq q$.

Proof. Let $\mu$ be a monomial of $P(f, g)$ which is determined by the following two properties:

- $J=\operatorname{deg}_{g}(\mu)$ is the largest possible.

- Among the monomials of $P(f, g)$ with $\operatorname{deg}_{g}$ equal to $J, \mu$ is the largest in the lexicographic ordering given by $f>>g$. 
If $J \leq q$ our claim is obviously correct since $\operatorname{deg}_{s}$ for all monomials of $Q(t, s)$ is at most $J$. So assume that $J>q$. Let $I=\operatorname{deg}_{f}(\mu)$. Since $P(f, g)$ is $n, m$-homogeneous, $n I+m J=N$. If $I+J>2 q+1$ then

$N=n I+m J=n(I+J)+(m-n) J \geq n(2 q+2)+(m-n)(q+1)=(n+m)(q+1)$

and so $N /(n+m) \geq q+1$, which is impossible since $q$ is the integral part of $N /(n+m)$. So $I+J \leq 2 q+1$. (The reader is reminded that $m>n$.) Let $\mu=f^{i_{1}} g^{j_{1}} \ldots f^{i_{k}} g^{j_{k}}$. After the substitution it becomes

$$
\mathbf{m}=t^{n i_{1}}\left(t^{m}+s\right)^{j_{1}} \ldots t^{n i_{k}}\left(t^{m}+s\right)^{j_{k}} .
$$

We would like to find a monomial $v$ in $\mathbf{m}$ with $\operatorname{deg}_{s}(v) \leq q$ which cannot come from any other monomial of $P(f, g)$ after the substitution. Then $v$ cannot be cancelled out and is a monomial of $Q(t, s)$.

First let us obtain a monomial $\pi$ of $\mathbf{m}$ by the following rule. If $j_{r}=2 \sigma_{r}$ replace $\left(t^{m}+s\right)^{j_{r}}$ by $\left(s t^{m}\right)^{\sigma_{r}}$; if $j_{r}=2 \sigma_{r}+1$ replace $\left(t^{m}+s\right)^{j_{r}}$ by $\left(s t^{m}\right)^{\sigma_{r}} s$. Then $\operatorname{deg}_{s}(\pi) \leq$ $\sum_{r}\left[\left(j_{r}+1\right) / 2\right]=(J+k) / 2$. Since $i_{1} \geq 0$ and $i_{r}>0$ for $r>1$ it is clear that $I \geq k-1$. So $J+k \leq J+I+1 \leq 2 q+2$. Therefore $\operatorname{deg}_{s}(\pi) \leq q+1$.

If $\operatorname{deg}_{s}(\pi) \leq q$ take $v=\pi$. If $\operatorname{deg}_{s}(\pi)=q+1$ then $I+J=2 q+1$, all $j_{s}$ are odd, and $k=I+1$. Therefore $i_{1}=0, i_{2}=\cdots=i_{k}=1$. To obtain $v$ in this case replace $\left(s t^{m}\right)^{\sigma_{1}} s$ in $\pi$ by $\left(t^{m} s\right)^{\sigma_{1}} t^{m}$.

If $\operatorname{deg}_{s}(\pi)=q+1$ then $v=\left(t^{m} s\right)^{\sigma_{1}} t^{m+n}\left(s t^{m}\right)^{\sigma_{2}} s t^{n} \ldots\left(s t^{m}\right)^{\sigma_{k}} s$. If $\xi$ is a monomial of $P(f, g)$ and $v$ is contained in its image after the substitution then any $s$ appearing in $\nu$, any $t^{m}$ appearing as $s t^{m} s$, and the first $t^{m}$ correspond to $g$ in $\xi$. Also $t^{n}$ appearing as $s t^{n} s$ corresponds to $f$ in $\xi$. So the only ambiguity is $t^{m+n}$ which corresponds to either $g f$ or $f g$. In any case $\operatorname{deg}_{s}(\xi)=J$ and if instead of $g f$ appearing in $\mu$ we put $f g$ then $\xi$ is larger than $\mu$ in the lexicographic ordering contrary to our assumption on $\mu$.

If $\operatorname{deg}_{s}(\pi) \leq q$ then any $\left(s t^{m}\right)^{\sigma} s$ corresponds to $g^{2 \sigma+1}$ in $\xi$. Now, $\left(s t^{m}\right)^{\sigma} t^{i n} s=$ $\left(s t^{m}\right)^{\sigma-1} s t^{m+i n} s$ and corresponds to $g^{2 \sigma-1} f^{\tau} g f^{i-\tau} g$ for $0 \leq \tau \leq i$. As above this means that $\operatorname{deg}_{s}(\xi)=J$ and that the only choice possible is $\tau=0$ since otherwise $\xi>\mu$ in the lexicographic ordering.

The lemma is proved.

Estimate. Recall that $\operatorname{deg}(f)=n, \operatorname{deg}(g)=m, \operatorname{deg}(t)=1, \operatorname{deg}(s)=\operatorname{deg}([f, g])-$ $\operatorname{deg}(f)=\operatorname{deg}([f, g])-n \leq m, P(f, g)$ is $n, m$-homogeneous and $N=w_{n, m}(P(f, g))$. As we checked, $Q(t, s)=P\left(t^{n}, t^{m}+s\right)$ contains a monomial $v$ for which $\operatorname{deg}_{s}(v) \leq$ $N /(n+m)$. If $i=\operatorname{deg}_{t}(v)$ and $j=\operatorname{deg}_{s}(v)$ then $N=i+j m$ and $\operatorname{deg}(v)=i+$ $j(\operatorname{deg}([f, g])-n)$. So $\operatorname{deg}(v)=N+j(\operatorname{deg}([f, g])-n-m)$. Therefore

$$
\begin{aligned}
\operatorname{deg}(P(f, g)) & \geq \operatorname{deg}(v)) \geq N+\frac{N}{n+m}\left(\operatorname{deg}([f, g]-n-m)=\frac{\operatorname{deg}([f, g])}{n+m} N\right. \\
& =\frac{\operatorname{deg}([f, g])}{n+m} w_{n, m}(P) .
\end{aligned}
$$

Theorem 1.1 is proved. 
Example 3.1. Let $f=x^{n}, g=x^{m}+y, P=[x, y]^{k}$. Then

$$
\operatorname{deg}(P(f, g))=k(n+1)=\frac{\operatorname{deg}([f, g])}{\operatorname{deg}(f g)} w_{n, m}(P)
$$

shows that the bound in Theorem 1.1 cannot be improved.

Remark 3.2. If $n \mid m$ the estimate does not work. Take e.g. $f=x, g=x^{m}+y$, and $P=g-f^{m}$. Then $\operatorname{deg}(P(f, g))=1<\frac{2}{1+m} m$. This happens because the degree drop for $g-f^{m / n}$ in this case is larger than the degree drop of $[f, g]$.

\section{Degree estimate for polynomial algebras}

In this section $F[t, s]$ is a polynomial algebra of rank two. Recall that $f=t^{n}, g=t^{m}+s$, $P(f, g)$ is an $n, m$-homogeneous polynomial, and $N=w_{n, m}(P(f, g))$. We would like to estimate $\operatorname{deg}(P(f, g))$ if $\operatorname{deg}(t)=1, \operatorname{deg}(s)=\operatorname{deg}(d f \wedge d g)-\operatorname{deg}(f) \leq \operatorname{deg}(g)$, and deg is a weighted degree function on $F[t, s]$ determined by these weights. Let $d=(n, m)$ be the greatest common divisor of $n$ and $m$, and $n_{1}=n d^{-1}, m_{1}=m d^{-1}$. Put $f_{1}=g^{n_{1}}-$ $f^{m_{1}}$. Then $w_{n, m}\left(f_{1}\right)=n m_{1}=n_{1} m$. We can write $P(f, g)=\sum c_{i, j, k} f^{i} g^{j} f_{1}^{k}$ where $c_{i, j, k} \in F$ and $0 \leq i<m_{1}$. Since $P(f, g)$ is $n, m$-homogeneous, $i n+j m+k n_{1} m=N$ and does not depend on a summand. So $N-i n$ is divisible by $m$ and since $i<m_{1}$ we can conclude that $i$ is the same for all summands. Hence $P(f, g)=f^{i} \sum c_{j, k} g^{j} f_{1}^{k}$ where $0 \leq i<m_{1}$ and different summands have different $k$.

Clearly for all summands $k \leq N / n m_{1}$. So $\kappa=\min \left\{k \mid c_{j, k} \neq 0\right\}$ does not exceed $N / n m_{1}$. Take this summand $f^{i} g^{l} f_{1}^{\kappa}$. Consider $Q(t, s)=P\left(t^{n}, t^{m}+s\right)$. Then $Q(t, s)$ contains a monomial $\mu=t^{i n+\iota m+\kappa m\left(n_{1}-1\right)} s^{\kappa}$ with non-zero coefficient since $f_{1}=n_{1} t^{m\left(n_{1}-1\right)} s+\cdots$ and all other monomials of $Q(t, s)$ have larger $\operatorname{deg}_{s}$. Since deg is a weighted degree function on $F[t, s]$ we have $\operatorname{deg}(P(f, g))=\operatorname{deg}(Q(t, s)) \geq \operatorname{deg}(\mu)$. So

$$
\operatorname{deg}(P(f, g)) \geq i n+\iota m+\kappa m\left(n_{1}-1\right)+\kappa \operatorname{deg}(s)=N+\kappa(\operatorname{deg}(s)-m) .
$$

Since $\operatorname{deg}(s)-m \leq 0$ we conclude that

$\operatorname{deg}(P(f, g)) \geq N+\frac{N}{n m_{1}}(\operatorname{deg}(d f \wedge d g)-n-m)=N\left[1-\frac{n+m-\operatorname{deg}(d f \wedge d g)}{n m_{1}}\right]$,

which is the estimate of Proposition 1.2.

Acknowledgments. The authors are grateful to the Shanghai Institute for Advanced Studies of the University of Science and Technology of China and the Beijing International Center for Mathematical Research for warm hospitality during their visits when part of this project was carried out. They also thank George Bergman for kindly pointing out references [3, 4]. Finally, they would like to thank V. Drensky, P. Malcolmson, and V. Shpilrain for comments and suggestions.

The research of L. Makar-Limanov was partially supported by an NSA Grant. The research of Jie-Tai Yu was partially supported by an RGC-CERG Grant. 


\section{References}

[1] Abhyankar, S. S., Moh, T. T.: Embeddings of the line in the plane. J. Reine Angew. Math. 276, 148-166 (1975) Zbl 0332.14004 MR 0379502

[2] Bergman, G. M.: Centralizers in free associative algebras. Trans. Amer. Math. Soc. 137, 327344 (1969) Zbl 0175.31501 MR 0236208

[3] Bergman, G. M.: Conjugates and $n$th roots in Hahn-Laurent group rings. Bull. Malaysian Math. Soc. 1, 29-41 (1978) Zbl 0395.16002 MR 0506536

[4] Bergman, G. M.: Historical addendum to: "Conjugates and $n$th roots in Hahn-Laurent group rings”. Bull. Malaysian Math. Soc. 2, 41-42 (1979) Zbl 0409.16001 MR 0545801

[5] Hahn, H.: Über die nichtarchimedischen Grössensysteme. Sitzungsber. Math.-Natur. K1. Kaiserl. Akad. Wiss. 116, 601-655 (1907) JFM 38.0501.01

[6] Makar-Limanov, L.: On the groups of automorphisms of certain algebras. Dissertation, Moscow State Univ., Moscow (1970) (in Russian)

[7] Makar-Limanov, L.: Algebraically closed skew fields. J. Algebra 93, 117-135 (1985) Zbl 0558.16007 MR 0780486

[8] Mal'tsev, A. I.: On the embedding of group algebras in division algebras. Dokl. Akad. Nauk SSSR (N.S.) 60, 1499-1501 (1948) (in Russian) MR 0025457| Zbl 0034.30901

[9] Mikhalev, A. A., Shpilrain, V., Yu, J.-T.: Combinatorial Methods: Free Groups, Polynomials, and Free Algebras. CMS Books in Math. 19, Springer, New York (2004) Zbl 1039.16024 MR 2014326

[10] Moufang, R.: Einige Untersuchungen über geordnete Schiefkörper. J. Reine Angew. Math. 176, 203-223 (1937) Zbl 0015.34202

[11] Neumann, B. H.: On ordered division rings. Trans. Amer. Math. Soc. 66, 202-252 (1949) Zbl 0035.30401 MR 0032593

[12] Neumann, B. H.: On ordered groups. Amer. J. Math. 71, 1-18 (1949) Zbl 0031.34201 MR 0028312

[13] Shestakov, I. P., Umirbaev, U. U.: Poisson brackets and two-generated subalgebras of rings of polynomials. J. Amer. Math. Soc. 17, 181-196 (2004) Zbl 1044.17014 MR 2015333

[14] Shestakov, I. P., Umirbaev, U. U.: The tame and the wild automorphisms of polynomial rings in three variables. J. Amer. Math. Soc. 17, 197-220 (2004) Zbl 1056.14085 MR 2015334

[15] Shimbireva, H.: On the theory of partially ordered groups. Rec. Math. [Mat. Sbornik] N.S. 20 (62), 145-178 (1947) (in Russian, English summary). Zbl 0029.10301 MR 0020558

[16] Suzuki, M.: Propriétés topologiques des polynômes de deux variables complexes, et automorphismes algébriques de l'espace $C^{2}$. J. Math. Soc. Japan 26, 241-257 (1974) Zbl 0276.14001 MR 0338423

[17] Umirbaev, U. U.: Yu, J.-T.: The strong Nagata conjecture. Proc. Nat. Acad. Sci. U.S.A. 101, 4352-4355 (2004) Zbl 1065.13011 MR 2055075 